\title{
Summary Report of Working Group 3: High Gradient and Laser-Structure Based Acceleration
}

\author{
N. Solyak ${ }^{\mathrm{a}}$ and B. M. Cowan ${ }^{\mathrm{b}}$ \\ ${ }^{a}$ Fermi National Accelerator Laboratory \\ ${ }^{b}$ Tech-X Corporation, 5621 Arapahoe Ave., Boulder, CO 80303
}

\begin{abstract}
The charge for the working group on high gradient and laser-structure based acceleration was to assess the current challenges involved in developing an advanced accelerator based on electromagnetic structures, and survey stateof-the-art methods to address those challenges. The topics of more than 50 presentations in the working group covered a very broad range of issues, from ideas, theoretical models and simulations, to design and manufacturing of accelerating structures and, finally, experimental results on obtaining extremely high accelerating gradients in structures from conventional microwave frequency range up to $\mathrm{THz}$ and laser frequencies. Workshop discussion topics included advances in the understanding of the physics of breakdown and other phenomena, limiting high gradient performance of accelerating structures. New results presented in this workshop demonstrated significant progress in the fields of conventional vacuum structure-based acceleration, dielectric wakefield acceleration, and laser-structure acceleration.
\end{abstract}

Keywords: Accelerating structure, high gradient, breakdown, wakefield, dielectric wakefield acceleration, laser-driven acceleration

PACS: $41.75 . \mathrm{Lx} ;$ 41.20.Jb; 41.75.Jv

\section{HIGH GRADIENT AND BREAKDOWN STUDIES IN METALLIC STRUCTURES}

The development of high-gradient (HG), high-frequency accelerator structures has raised the possibility of a $\mathrm{TeV}$-scale linear collider for exploring particle physics at the energy frontier. Two proposed projects of a future linear collider, the so-called Next Linear Collider (NLC) and Compact Linear Collider (CLIC), are based on room temperature high gradient accelerating structures made of copper. These projects have triggered intense, systematic studies of gradient limitations caused by breakdown. Recently, research to study the limits of room temperature linacs has been stimulated by national and international collaborations, leading to new advances in the state of the art. The studies were focused on incremental improvement of existing design, construction and surface preparation techniques of practical, full-scale accelerating structures. Electron linacs based on this technology have very wide application in other fields, such as cancer therapy, gamma production, and security. In this session the presentations provided an overview of recent experimental results on breakdown and designs proposed to improve performance of HG structures.

A. Grudiev of CERN overviewed recent CERN progress in high gradient investigations. These studies were done by a wide international collaboration including CERN, SLAC, and KEK. This collaboration built and tested more than 30 different types of accelerating structures with different geometries. It was found that the surface field $E$, RF pulse duration $t$, and breakdown rate $B D R$ can be fitted by the relation that $E^{30} t^{6} / B D R$ is constant. Nevertheless, the scatter of measured accelerating gradients in all investigated structures is fairly large (factor of $~ 6$ ) and depends on group velocity, geometry and other parameters. It was proposed to use new constraint parameter, which better reflects the physics of breakdown: $s=\operatorname{Re}(P)+\operatorname{Im}(P) / 6$, where $P$ is Poynting vector in accelerating structure. The underlying physics is the fact that power needed for field emission comes from the RF power traveling in structure. All available data have significantly less scatter using this parameter instead of the accelerating gradient. Some of these studies were reported in previous papers. Now, a new constraint together with other constraints, including temperature rise due to pulsed heating, was applied in designing of the second generation of the CLIC accelerating structures. 
V. Dolgashev of SLAC presented work on studies of RF breakdown physics in normal conducting cavities. It was observed that after the initial conditioning, the dependence of the breakdown rate on pulse duration and RF power is remarkably reproducible for different structures of the same geometry and material. It allows one to interpolate breakdown performance data to new geometries. The comparison of traveling wave (TW) and standing wave (SW) structures at low breakdown rate $(<10$ per hour) shows that in spite of the very similar behavior of the short SW and long, low group velocity TW structures, TW structures sustain typically $\sim 20-30 \%$ lower gradient and about 2 times lower peak pulsed heating. One of the possible reasons is the difference in the way RF power is absorbed in a structure after breakdown. It is also possible that the difference is related to the different length of the structures: one-cell SW structures vs. 18 or more cell TW structures. To address all these questions, the SLAC team, in collaboration with other institutions, is planning a series of new experimental studies, including investigation of breakdown phenomena at low temperatures (100 K or below) and at room temperature by using advanced in-situ visual diagnostics to see evolution of surface microscopic pictures for different types of structures.

The maximum achievable accelerating gradient is defined not only by the geometry, but also by the material of the cavity. In the past, a number of studies were done to understand the influence of the material on cavity performance. In this workshop, J. Haimson of Haimson Research Corporation presented his work on design and fabrication of the $17 \mathrm{GHz}, 22$-cell accelerating structure with a molybdenum surface in the dark current interception regions, brazed to copper. This structure is planned for testing in the MIT resonant ring at $17 \mathrm{GHz}$. The results will be compared with results received in previous tests for all-copper and Cu/SST structures with the same geometry. Dr. Haimson also presented the status of the design of a new $11 \mathrm{GHz}$ resonant ring being built for testing of the CLIC type structures at SLAC.

F. Wang of SLAC presented work on studies of limiting effects in X-Band accelerator structures. The major focus of his talk was analysis of experimental studies carried out to understand the effect of pulsed heating on the breakdown rate (BDR) in the cavity. In experiments with pre-heating of the cavity surface by a series of RF pulses before the main pulse was applied, it was discovered that the BDR is very sensitive to surface temperature. For the same fields in a cavity and the same pulse duration, increasing the surface temperature by $\sim 10$ degrees in a preheated cavity leads to an increase of a few times in the BDR. The measured breakdown rate of the detuned CLIC structure TD18 was two orders of magnitude higher than in the cylindrically symmetric structure T18 with the same geometry, which could be related to different pulsed heating. To continue studies of the pulsed heating effect on cavity performance, SLAC has designed a dual-mode cavity, which will be used to study breakdown by varying the combination of $\mathbf{E}$-field and $\mathbf{H}$-field.

A photonic bandgap ( $\mathrm{PBG}$ ) accelerating structure was proposed to cure problems with higher-order mode (HOM) damping. A specially designed array of rods with a regular structure traps an accelerating mode in the cavity, while most other modes radiate out of the cavity. B. Munroe of MIT presented a talk on breakdown studies of PBG accelerator structures at 11 and $17 \mathrm{GHz}$. An $11 \mathrm{GHz}$ one-cell PBG cavity tested at SLAC showed >100 $\mathrm{MV} / \mathrm{m}$ gradient without damage in the high electric field area, but showed significant damage in the high $\mathbf{H}$-field area - the surface of the rods - due to high pulsed heating. In a new design, the MIT team proposes to modify the shape of the rod, using elliptical irises to reduce the pulse temperature rise below $50 \mathrm{~K}$ (instead of $>100 \mathrm{~K}$ ). The new design is planned for testing at $17 \mathrm{GHz}$ on the new MIT test stand being built.

New ideas on how to increase accelerating gradient, based on using multimode cavities with harmonically related frequencies, were presented in the talks of S. Kuzikov (shown by J. Hirshfield) and Y. Jiang of Omega-P, Inc. Multimode structures can be excited by a drive beam or by independent RF power sources for each frequency used. Since the accelerated beam will see all the modes in phase, this allows an increase in the ratio of the accelerating to the surface field. Another advantage of a multimode cavity is the reduction of the exposure time of the peak field on the cavity surface. Y. Jiang also presented the design of a multi-mode cavity with asymmetric longitudinal geometry to explore the idea that a cavity can sustain higher surface electric fields if the sign of this field prevents emission of electrons (anode-cathode effect). It is expected that it is possible to increase the accelerating gradient in a multimode cavity by up to $50-70 \%$ compared to a single mode cavity. Finally, three designs of the cavity were proposed for testing to prove all these ideas. In another talk, Y. Jiang presented the idea to use a structure with alternatively detuned cells for a two-beam accelerator scheme to provide a high transformer ratio (well above 2) with reasonable energy transfer efficiency. In a numerical example, an accelerating gradient of over $150 \mathrm{MV} / \mathrm{m}$ is predicted for a cavity detuning of $\Delta \omega / \omega=0.9 \times 10^{-3}$, with a transformer ratio of 13 and a beam-to-beam power transfer efficiency of $60 \%$ for a drive beam of $\sim 100 \mathrm{~A}$ and an accelerated beam of $4.8 \mathrm{~A}$. To minimize transients in detuned cavity excitation, a few schemes were proposed using a shaped pre-pulse of RF power to cancel beating. 


\section{BREAKDOWN IN DIELECTRIC AND SUPERCONDUCTING STRUCTURES}

Application of dielectric materials in accelerators has a long history. In the last few years one can see growing interest in $\mathrm{HG}$ accelerating structures based on them. This interest is triggered by progress in improving dielectric performance and development of new materials. A. Kanareykin of Euclid TechLabs presented recent results on development and experimental testing of advanced dielectric materials, for use with pulsed high power from microwave frequencies up to the $\mathrm{THz}$ frequency range, and specially designed for accelerator applications. The list of these materials includes low loss microwave ceramic and quartz and chemical vapor deposition (CVD) diamond that has been considered for a dielectric based accelerating structure, and nonlinear ferroelectrics for RF tuning and phase shifting components to provide fast electronic control. Accelerating gradients of $\sim 100 \mathrm{MV} / \mathrm{m}$ and $\sim 1 \mathrm{GV} / \mathrm{m}$ have been demonstrated for microwave and $\mathrm{THz}$ dielectric structures, respectively. The conclusion of his talk is that electrical and mechanical properties of diamond make it an ideal candidate material for use in dielectric RF structures.

Dielectric-loaded accelerating (DLA) structures have number of advantages vs. metallic structures: simple geometry, larger aperture, and potentially the ability to provide higher gradient, limited by breakdown in dielectric materials. To study performance of DLA structures for different dielectric materials, a magnicon facility with available power of $\sim 20 \mathrm{MW}$ at X-band is used. The status and the results of this work were presented by S. Gold of NRL in his talk, "Development of X-band dielectric-loaded accelerating structures." Up until today, a few dielectric materials were tested in DLA structures, and the achieved gradient of $\sim 15 \mathrm{MV} / \mathrm{m}$ was limited by breakdown in the joint between ceramic pieces, not in the dielectric. Multipactoring (MP) is still an issue for dielectric structures, and future experiments plan to use coating and grooving to suppress multipactoring. The geometry of the DLA also has to be modified to get higher gradients for available power.

Y. Jiang of Yale University presented work on a CVD diamond RF breakdown experiment. This experiment applies strong tangential RF electric fields of up to $500 \mathrm{MV} / \mathrm{m}$ to the surface of CVD diamond. To date, $450 \mathrm{~kW}$ of power from a Ka-band magnicon, corresponding to $\sim 150 \mathrm{MV} / \mathrm{m}$ surface field, has been applied with a hydrogenated sample in place without evidence of breakdown.

Work on multipactoring modeling and simulation for DLA structures was presented by O. Sinitsyn of the University of Maryland. MP simulations, done for alumina and quartz materials, predicted significant power loss along the structure. Simulations are in reasonably good agreement with experimental data obtained at ANL and NRL.

New ideas and experimental studies on high gradients in superconducting (SC) cavities and materials were discussed in the working group in two talks. J. Guo of SLAC presented a system, which was designed for testing superconducting materials at low cryogenic temperatures, using an X-band, high- $Q\left(\sim 10^{5}\right)$ copper cavity. The magnetic field in the cavity reaches its maximum value on the flat surface, which is made of the SC material (niobium, $\mathrm{MgB}_{2}$, multilayer, high-T superconductors, etc.) under test. RF pulsed power from a $50 \mathrm{MW}$ klystron is able to provide $>200 \mathrm{mT}$ magnetic field on the surface of the samples. The first results of testing a few samples clearly detect transition from the superconducting to the normal conducting state at the field at which quench happened. This is a promising test bed for superconducting RF (SRF) material investigations. N. Solyak and V. Yakovlev of Fermilab presented the design of a niobium TW superconducting structure and the first results of high gradient tests of a single-cell prototype, built by Euclid TechLabs. The maximum magnetic field in the tests, of $\sim 120 \mathrm{mT}$, achieved in the feedback waveguide is well above the limit required for high gradient operation of the proposed structure. There is a plan to build and test a multi-cell superconducting structure to demonstrate the advantages of the proposed approach.

\section{BREAKDOWN THEORY}

One session was organized to overview and discuss new theoretical models of breakdown phenomena in metallic and dielectric structures, presented in a few talks from the University of Maryland theory group (G. Nusinovich, W. Zhu, and D. Kashin). They have analyzed two effects: the role of micron-scale protrusions, and ion bombardment. The modeling shows that protrusions originated by micro-cracks increase the local electric and magnetic fields, thus increasing by a factor of 2 the temperature rise from pulsed heating, as well as resulting in field emission. Walls with protrusions experience large mechanical stresses. Simulations show that the effect of ion bombardment is less important than expected. 
Applying external magnetic fields on an RF cavity may play a significant role in the reduction of accelerating gradient. This is an important issue for cooling sections of a muon collider, where high magnetic fields are required to focus the beam.

$\mathrm{S}$. Veitzer of Tech-X Corporation presented the modeling of a HG pillbox cavity at $805 \mathrm{MHz}$ with an applied magnetic field of different strengths and orientations. The effects of field emission and space charge were simulated using 3D code VORPAL. It was shown that the magnetic field has a strong effect on electron orbits and some orientations can confine secondary electrons near the cavity walls, creating more secondary electrons. D. Stratakis of UCLA presented the idea of a magnetically insulated cavity for muon cooling. This concept involves designing an RF cavity with walls that are parallel to the contour lines of the external magnetic fields, thereby redirecting field-emitted electrons back to the cavity surface before they gain energy from the RF electric field. He presented the design of such a system and plans for testing this concept. The following discussion in a joint session of the high gradient and muon groups was productive to understand potential problems.

\section{DEVELOPMENT OF RF HIGH POWER COMPONENTS}

A. Murokh of RadiaBeam Techologies, LLC presented work on an ultra-high gradient S-band linac for laboratory and industrial applications. The goal of this work is to develop a compact ( $\sim 0.6 \mathrm{~m})$ copper accelerating structure (the DECA structure), and demonstrate $50 \mathrm{MV} / \mathrm{m}$ accelerating gradient. The authors presented an optimized RF design, thermal and mechanical stress analysis, and plans for prototyping and testing.

A. Vikharev of the Institute of Applied Physics, Russia and Omega-P, Inc. presented work on development of a high power active microwave switch, employing electron beam triggering for use in pulse compression systems. In the proposed concept the electron beam detunes the switching cavity out of resonance, triggering mode conversion in a storage resonator. At low power tests at $11.424 \mathrm{GHz}$, the achieved parameters include a power gain of 20 with a switching time of $\sim 25 \mathrm{~ns}$ and good stability. A high power test is planned using a magnicon as a high power Xband source.

J. Neilson of SLAC presented the conceptual layout and design of an RF feed system for standing wave accelerating structures. SW structures have advantages over TW structures, providing robustly higher gradient. The RF feed system is designed to distribute power from a klystron to a module of 18 cells, feeding cells in parallel through 4 ports per cell. The components of the proposed design are under construction.

\section{DIELECTRIC WAKEFIELD ACCELERATION}

Dielectric wakefield acceleration (DWA) consists of passing a high-charge particle bunch through a dielectric waveguide to excite strong electromagnetic fields, and then using those fields to accelerate a lower-charge witness bunch. This method is being explored over a frequency range from the RF to the THz. A key challenge in designing DWA based accelerators is to improve the transformer ratio beyond the conventional limitation of 2 . S. Shchelkunov of Yale presented work on a two-channel DWA, in which the dielectric-lined channel is separated by a dielectric slab into a large channel, for the drive bunch, and a smaller, higher-impedance channel for the witness bunch. These structures will be tested at the Argonne Wakefield Accelerator (AWA) facility, to study the basic physics and demonstrate a transformer ratio of $\sim 12$. T. Marshall of Columbia presented work on a coaxial two-channel design, which includes an inner dielectric cylinder suspended within the waveguide. The drive bunch propagates outside, and the witness bunch inside, the inner cylinder. Simulations showed a peak accelerating gradient of $1.5 \mathrm{GeV} / \mathrm{m}$ and a transformer ratio of $\sim 6$. G. Sotnikov of the Kharkov Institute conducted simulations of bunch stability in the coaxial structure, finding that in cm-scale devices, misalignments of 5-10\% were acceptable, but in $\mathrm{mm}$-scale devices, the tolerance was much smaller, $\sim 5 \mu \mathrm{m}$.

The upcoming FACET facility at SLAC presents significant opportunities for investigating dielectric wakefield acceleration in the THz regime. J. Rosenzweig of UCLA presented plans and objectives for THz DWA experiments at FACET. These include a detailed study of breakdown, and explorations of a variety of structure materials and geometries. S. Antipov of Euclid TechLabs presented simulations of beam break-up in a diamond-based DWA operating in the $\mathrm{THz}$ regime with parameters relevant to upcoming experiments at FACET. It was shown that micron-scale offsets of the beam position from axis resulted in $10 \%$ beam loss within centimeters of propagation. A. Kanareykin of Euclid TechLabs presented possible diamond-based THz structure designs for FACET experiments, including planar and cylindrical geometries, showing simulations of gradient and beam break-up for both cases. He also discussed fabrication issues, as well as the possibility of a thermo-conducting cladding. 
C. Jing of Euclid TechLabs presented work on power extractors based on dielectric waveguides. Tens of MW power extraction has been demonstrated at AWA in C-band and Ka-band, with hundreds of MW expected possible with the forthcoming AWA upgrade. Work is also beginning on X-band power extraction. W. Gai of ANL presented a concept of a DWA-based linear collider. The concept includes $1 \mathrm{GeV}$ drive beams, each driving a $100 \mathrm{GeV}$ module with a $200-300 \mathrm{MeV} / \mathrm{m}$ gradient. M. Conde of ANL discussed the upcoming AWA upgrade, which includes capabilities for bunch trains of 32 bunches of $60 \mathrm{nC}$ each, and a beam energy of $75 \mathrm{MeV}$. This should enable demonstration of 300-500 MeV/m accelerating gradient in DWA structures, energy gains of $100 \mathrm{MeV}$, and GW level power extraction.

\section{SIMULATIONS}

C. Nieter of Tech-X Corp. presented VORPAL simulations of multipacting in RF structures. He discussed the secondary electron yield model used, and showed good agreement with experimental data. Detailed simulations of multipacting in the Jefferson Lab crab cavity were described. J. DeFord of AWR presented multipacting capability in the Analyst code, including the particle trajectory integration method and the secondary electron yield model. A number of simulation examples were shown. C. Bauer of the University of Colorado presented an algorithm for simulating dielectric materials with sharp interfaces using the finite-difference time-domain method. He showed that the algorithm achieves second-order convergence of frequencies. A. Candel of SLAC presented large-scale simulations of CLIC two-beam accelerator structures using the time-domain T3P code. The finite element discretization and boundary condition capabilities were discussed. Examples of wakefield damping and power extraction were shown.

\section{NOVEL MATERIALS AND GEOMETRIES}

A number of advanced structure concepts employ novel properties of a bulk medium. These properties can be from a bulk material, or be engineered by introducing a geometry structured on the scale of the operating wavelength. G. Shvets of the University of Texas discussed a surface-wave accelerator composed of silicon Carbide $(\mathrm{SiC})$. This structure takes advantage of the negative permittivity property of $\mathrm{SiC}$ in the far infrared regime. The presentation explored both laser-driven and beam-driven operation of a $\mathrm{SiC}$ structure with a prism geometry. Simulation and experimental observation of accelerating and deflecting wakes were shown. M. Shapiro of MIT showed work on complementary metamaterial accelerator structures composed of arrays of split-ring resonators which have negative effective index. Simulations of the negative-index TM accelerating mode were shown as well as plans for experimental test.

C. Jing of Euclid TechLabs showed experimental studies of X-band metallic photonic bandgap (PBG) structures at the AWA. These studies characterized the modes in the structure, finding that the accelerating mode has a $Q 10$ times higher than the higher order modes (HOMs), and also showed $\sim 30 \mathrm{MV} / \mathrm{m}$ gradient in a collinear acceleration test. A. Cook of MIT demonstrated the concept of HOM damping in a metallic PBG structure by removing rows of rods. This significantly reduces the diffractive $Q$ of the HOMs while leaving the $Q$ of the accelerating mode nearly intact.

\section{LASER-STRUCTURE BASED ACCELERATION}

Laser-driven accelerator structures can be classified into two broad categories: transversely driven structures, in which the drive laser is incident on the structure from the side, and excites the length of structure which it illuminates; and waveguide structures, in which an accelerating mode co-propagates with the particle beam in a waveguide. Among the former type of structure, A. Mikhailichenko of Cornell presented his work on a traveling laser focus concept, in which a laser beam is swept along a length of the accelerator. He also discussed some fundamental limitations of accelerator schemes. Another example of a transversely driven structure is the MicroAccelerator Platform (MAP), under development by a UCLA/Manhattanville College/RadiaBeam collaboration. R. Yoder of Manhattanville gave an overview of that effort. He presented the design concept, some simulation and fabrication results, and plans for experimental tests at the E163 facility at SLAC. He also discussed the concept of a micro-scale injector based on a pyroelectric crystal. J. McNeur of UCLA presented detailed simulation studies of the MAP structure. These included computation of the resonant fields, effects of different materials, studies of fabrication error tolerance, simulation of low $\beta$ structure concepts, and particle dynamics. J. Zhou of UCLA 
presented work on fabrication of the MAP structure. This includes deposition of the multilayer dielectric stack, and electron beam or focused ion beam lithography to create the coupling slots.

Studies of laser-driven waveguide structures based on PBG geometries were presented by the SLAC/Stanford/Tech-X collaboration. J. England of SLAC presented experimental plans for investigating PBG fiber structures at E163. He discussed the challenge of focusing the electron bunch through the $10 \mu \mathrm{m}$ aperture of the fiber core. J. Ng of SLAC showed simulation studies of wakefields in Bragg fibers. The short-range wakes appear to be manageable; work on long-range wakes is ongoing. R. Noble of SLAC presented computations of tachyon accelerating modes in PBG fibers - those with phase velocity $v_{p}$ close to, but slightly exceeding, $c$. He showed that these fast-wave modes tend to have better confinement in the fiber core than surface modes with $v_{p}=c$. Custom geometries being fabricated by Incom, Inc. were studied. C. Ng of SLAC presented computations of farfield radiation patterns from a PBG fiber, showing that a significant fraction of the power is radiated in 6 localized lobes. This information could potentially be used to couple power efficiently into such a structure. B. Cowan of Tech-X presented simulations of power couplers in a three-dimensional structure based on the woodpile PBG lattice. He showed that adjustment of individual rods near the coupler could yield a significant improvement in coupling efficiency, reaching over 95\%. C. McGuinness of SLAC described work on fabrication of woodpile-based structures. This included successful fabrication of 8 and 9 layer structures. He also showed spectroscopic measurements demonstrating experimental confirmation of the photonic band gap. B. Cowan of Tech-X presented studies of fabrication error in woodpile structures. These included measurements of the fabrication error of test structures, and simulations using parameters from those measurements to describe the effect of realistic fabrication error on accelerator parameters.

\section{ACKNOWLEDGMENTS}

We thank the participants in the working group for contributing the material summarized here, and for productive workshop discussion exploring these topics. References and acknowledgements for the work described herein can be found in the respective papers in these proceedings. 